\title{
Smartphone Apps in Graduate Medical Education Virtual Recruitment During the COVID-19 Pandemic
}

\author{
Neeral L. Shah ${ }^{1}$ (D) Jennifer B. Miller ${ }^{1} \cdot$ Mohammad Bilal $^{2} \cdot$ Brijen Shah $^{3}$ \\ Received: 14 January 2021 / Accepted: 3 February 2021 / Published online: 9 February 2021 \\ (C) The Author(s), under exclusive licence to Springer Science+Business Media, LLC part of Springer Nature 2021
}

\begin{abstract}
The COVID-19 pandemic required a shift of graduate medical education recruitment to a virtual format. In order to share information and insight into the culture of our program with applicants, we created a smartphone app for those that were invited for an interview. By collecting the analytics of the app, we were able to follow trends in the timing of applicants downloading the app, their viewing histories, and when information was accessed. The app was mostly downloaded at the time of the interview invite or $48 \mathrm{~h}$ prior to the interview day. Around the interview day, applicants tended to access trainee profiles, faculty profiles, and videos about the program and community. Closer to the rank list due date, training information, the graduate medical education (GME) documents, and the diversity and wellness initiatives seemed to have more activity. This analysis from the use of a smartphone app in virtual recruitment gives insight into the use of a smartphone app by applicants, and the information that they find useful during the process.
\end{abstract}

Keywords Smartphone app · Recruitment · Graduate medical education $\cdot$ Technology

\section{Introduction}

The COVID-19 pandemic impacted several aspects of graduate medical education including moving the fellowship and residency interview season to a virtual format [1-3]. Through a nationwide video conference call between gastroenterology fellowship program directors, the authors of this article brainstormed ideas. We sought to create an appealing virtual experience and incorporate innovative techniques to ensure a user-friendly process for applicants [4]. One strategy was the development of a smartphone application (app) to share in-depth information with applicants invited to

This article is part of the Topical Collection on Education \& Training

Neeral L. Shah

neeral.shah@virginia.edu

1 Division of Gastroenterology and Hepatology, University of Virginia, PO Box 800708, West Complex, Charlottesville, VA 22908, USA

2 Division of Gastroenterology, Minneapolis VA Medical Center, University of Minnesota, Minneapolis, MN, USA

3 Henry D. Janowitz Division of Gastroenterology, Department of Medicine, Icahn School of Medicine at Mount Sinai, New York, NY, USA interview. We aimed to evaluate the incorporation of a smartphone app on the virtual GI fellowship interview season.

\section{Methods}

\section{Smartphone app design}

Planning involved choosing an application to design our program's smartphone app. The web-based app design program provided multiple templates for individual pages allowing customization, editing portability, and rapid update publishing. Cost was a major factor in our decision. Pricing for an app ranged from $\$ 300$ to $\$ 600$ per year.

\section{Smartphone app content}

During the design process, 12 fellows and 10 faculty provided suggestions as test users. Based on feedback, it was evident the app was a dynamic tool, hence, it was geared to complement the program website. To create a connection to our trainees, current fellows recorded an informal video tour, which oriented applicants to the hospital and work spaces. The applicants could view a welcome video from the program 
director, faculty interviews, diversity information, and training details.

\section{Analytics and surveys}

We used analytics of the app to track use (downloads, views, individual page traffic). This information helped us to understand when the applicants utilized the app and what pages were used most frequently. Applicants had an option to complete a pre-interview survey to assist with feedback on the functionality of the app. A post-interview survey was sent to the applicants a day prior to the rank list due date.

\section{Results}

\section{Usability and views}

There were three time periods that applicants downloaded the app. About 23\% (9/40) downloaded the app within $48 \mathrm{~h}$ of receiving the interview invite. Seventeen percent (7 of 40) downloaded the app within 10 days of receiving the invite, while, the remaining 60\% (24/40) downloaded the app within the $48 \mathrm{~h}$ before interviewing. (Figure 1) Peak views of the app occurred the afternoon prior to an interview day (between $4 \mathrm{pm}-9 \mathrm{pm}$ ) and immediately after the interview (between $1 \mathrm{pm}-8 \mathrm{pm}$ ). The top pages of interest were: GI Fellows (599 views), GI faculty (480 views), Program Leadership (343 views), and Interview Day (182 views). (Table 1).

\section{Feedback from applicants}

Of the 40 applicants interviewed, $25(65 \%)$ responded to the pre-interview survey. Emerging themes from the free text responses regarding the most useful aspects included videos that provided insight into the culture (32\%), program training information (32\%), and program director's welcome video $(16 \%)$. Information that applicants felt was missing were biographical information about current fellows/faculty (32\%), fellow/faculty contact information (12\%), research projects (4\%), and post-training career information (4\%).

After the final interview day, we tracked the app use until the day prior to rank lists were due. Views of the app demonstrated two peaks after completion of interviews. The first peak (seven views) occurred ten days after the last interview day. Over the two weeks prior to the rank list due date, we noted an upward linear trend of app views: two weeks prior to the due date - two views, one week prior - six views, and the day prior - eleven views.

The post-interview survey was done prior to the rank list due date. We received 15 responses (38\%) from 40 applicants. Of particular interest, was the applicant direct comparison of an app to web pages. (Table 2) Respondents indicated that they were $53 \%$ more likely to review the program information included on the app than on the web. Additionally, $86 \%$ found the app slightly easier or much easier to use than a web page.

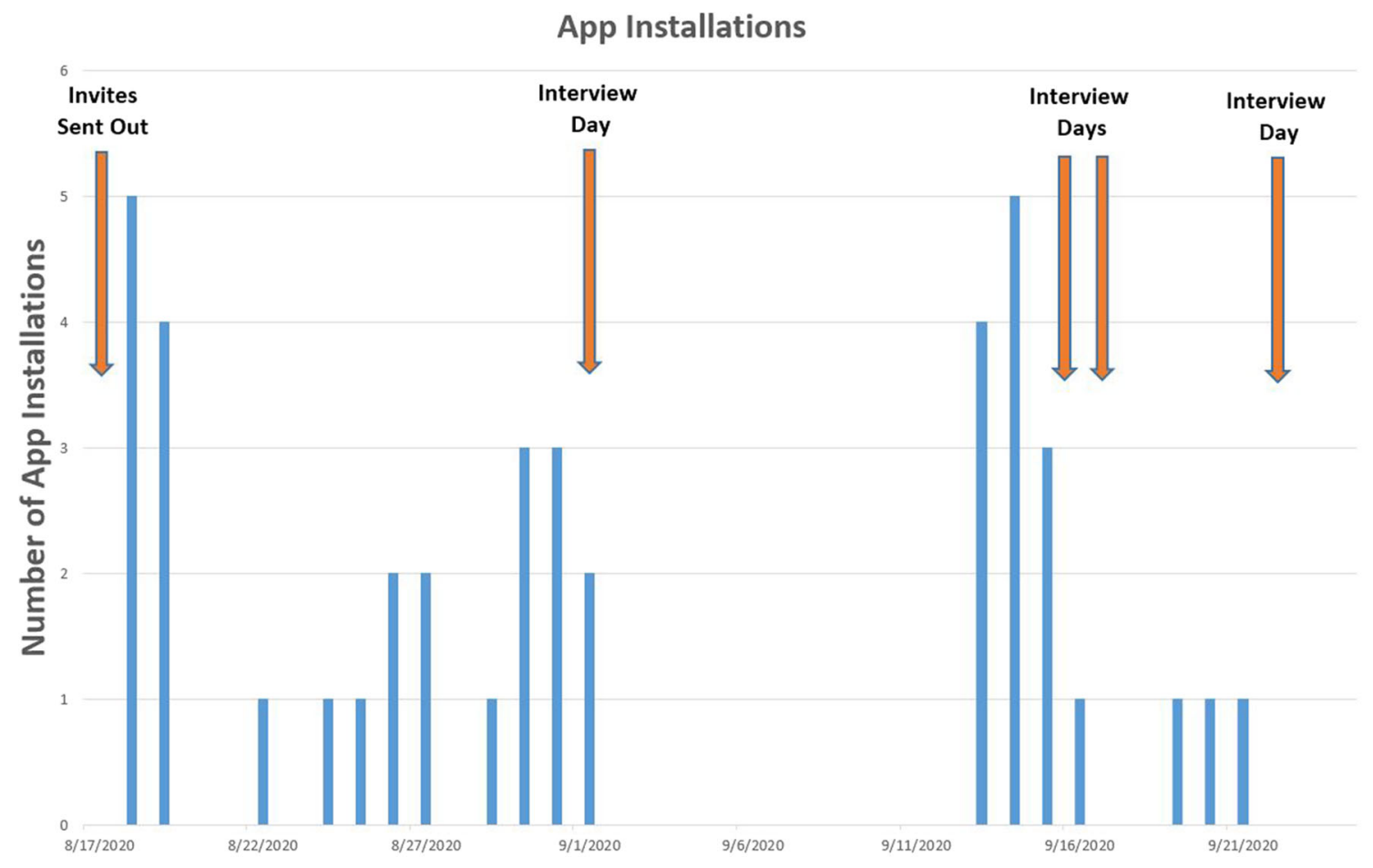

Fig. 1 App installations by date during interview season 
Table 1 Page views by applicants on smartphone app around the interview day

\begin{tabular}{ll}
\hline App Page & Views \\
\hline Current GI Fellows & 599 \\
Current GI Faculty & 480 \\
Program Leadership & 343 \\
Interview Day Schedule & 182 \\
GI Videos & 100 \\
Training Information & 98 \\
Endoscopy Training & 74 \\
1st Year Simulation Day & 67 \\
Welcome Page & 65 \\
Charlottesville Living & 54 \\
Fellows QI Projects & 54 \\
Newsletters & 53 \\
Interview Help & 50 \\
UVA GME, Diversity, Wellness & 47 \\
UVA Videos & 47 \\
GME Documents & 46 \\
Contact Information & 42 \\
\hline
\end{tabular}

None of the respondents thought the web was easier to use. When comparing content, $80 \%$ found the app content of higher or slightly more value than information on the web. Reflecting on the most useful pages of the app, program information was indicated by 53\%, and the GI videos, fellows' profiles, and fellow newsletters /QI projects were chosen by $13 \%$ of respondents. Eighty seven percent of respondents indicated a preference of having an app in addition to a website, but $87 \%$ were also opposed to all aspects of the interview to run solely through the app. Finally, 33\% indicated that the app has no effect on their rank list, but $47 \%$ reported a moderate effect on their final rankings.

The overall themes from free text responses indicated applicants felt the app was a good gesture to the commitment of recruitment (13\%), and provided easy access to the program information (13\%). However, some felt the app was too small on the phone, and that there is a need for emails as a method of record keeping (7\%).

\section{Conclusions}

Overall, applicants praised the easy usability, readily and quickly obtainable program information, and uniqueness of the app. The ability to access information on a phone, as opposed to using a web browser, was universally appealing. Program information on the app was accessed after invites were sent and within the $48 \mathrm{~h}$ prior to the interview day. To our knowledge, this is the first time this metric has been tracked from applicants. This timing should be considered to ensure information is up to date, and to consider pushing alerts or content prior to the interview day. As applicants envision their future workplace, personnel pages with fellows, faculty and leadership videos seemed to provide the most information, and therefore should be easily accessible to applicants prior to their interview.

Conversely, in the post-interview period, the more commonly viewed pages included training information, the graduate medical education (GME) documents, and the diversity and wellness initiatives. We assume this is due to applicants directly comparing aspects of multiple programs prior to submitting rank lists.

Finally, almost half of the applicants reported the app had a moderate effect on their rank list. We postulate that having program information readily available at the time of making rank list is helpful [5]. The interview trail can be long and it can be difficult to remember the fine details of each program. Furthermore, divisional websites and the traditional "interview day program folders" can be challenging to navigate, so having an app focused on the program provides an important resource.

By integrating new technological methods to enhance the experience, we found it is possible to carry out a successful virtual interview day. The addition of a smartphone app provides an innovative source of information for applicants. Many programs already utilize program websites [6, 7], which may not add additional cost to the virtual process, however the accessibility to make changes and flexibility to organize information is often limited by institutional barriers and approvals. Further, we were able to provide more personal information on the app (i.e. videos of the fellows, pictures from social events) that we would not include on a publicly available

Table 2 Applicant comparison of a program app vs. webpage

Compare the app to webpages by rating the ease of use?

\begin{tabular}{|c|c|c|c|c|}
\hline $\begin{array}{l}\text { App much easier } \\
53.3 \%\end{array}$ & $\begin{array}{l}\text { App slightly easier } \\
33.3 \%\end{array}$ & $\begin{array}{l}\text { No difference } \\
13.3 \%\end{array}$ & $\begin{array}{l}\text { Webpage slightly easier } \\
0 \%\end{array}$ & $\begin{array}{l}\text { Webpage much easier } \\
0 \%\end{array}$ \\
\hline \multicolumn{5}{|c|}{ Compare the app to webpages by the values of content provided? } \\
\hline $\begin{array}{l}\text { App content of higher value } \\
33.3 \%\end{array}$ & $\begin{array}{l}\text { App content slightly more value } \\
46.7 \%\end{array}$ & $\begin{array}{l}\text { No difference } \\
20 \%\end{array}$ & $\begin{array}{l}\text { Webpage of slightly more value } \\
0 \%\end{array}$ & $\begin{array}{l}\text { Webpage of higher value } \\
0 \%\end{array}$ \\
\hline
\end{tabular}


webpage. While there are variations in each program's resources, our surveys show that providing applicants readily accessible and organized program information may help with rank list decisions. Despite the challenging times our graduate medical education community faces, embracing new methods such as a smartphone app can enhance the interview experience. A continual examination of this process will be necessary as some combination of virtual and face to face interviews will likely be the way recruitment is conducted in the upcoming years. Future direction may include the identification of one or a few app development programs to be used by program directors, which may provide uniformity for applicants entering this process. Moreover, based on feedback from applicants each year, future versions of the app can be updated easily to include information that will be useful to applicants.

Code availability (software application or custom code) N/A

Authors' contributions NS - concept, writing, editing, JB - concept, writing, editing, $\mathrm{MB}$ - editing, $\mathrm{BS}$ - editing.

Data availability Available upon request.

Declarations Conflict of Interest: All authors have no financial or non-financial conflicts of interest with this manuscript.

Ethical approval This article does not contain any studies with human participants or animals performed by any of the authors.
Informed consent N/A

Conflict of interest None.

\section{References}

1. Mallepally N, Bilal M, Hernandez-Barco YG, et al. The New Virtual Reality: How COVID-19 Will Affect the Gastroenterology and Hepatology Fellowship Match. Dig Dis Sci 2020;65:2164-2168.

2. Clarke K, Bilal M, Sánchez-Luna SA, et al. Impact of COVID-19 Pandemic on Training: Global Perceptions of Gastroenterology and Hepatology Fellows in the USA. Dig Dis Sci 2020:1-5.

3. Gabrielson AT, Kohn JR, Sparks HT, et al. Proposed Changes to the 2021 Residency Application Process in the Wake of COVID-19. Acad Med 2020.

4. Jones RE, Abdelfattah KR. Virtual Interviews in the Era of COVID19: A Primer for Applicants. J Surg Educ 2020.

5. Gaeta TJ, Birkhahn RH, Lamont D, et al. Aspects of residency programs' web sites important to student applicants. Acad Emerg Med 2005; 12:89-92.

6. Ahmed SA, Hyman C, Eltorai AEM, et al. Evaluation of Integrated Interventional Radiology Residency Websites. R I Med J (2013) 2019;102:19-23.

7. Sherman NC, J CS, A MK, et al. The Content and Accessibility of Orthopaedic Residency Program Websites. JB JS Open Access $2020 ; 5$.

Publisher's Note Springer Nature remains neutral with regard to jurisdictional claims in published maps and institutional affiliations. 